Atsushi Namba • Satoko Abe • Hideichi Shinkawa

William J. Kimberling • Shin-ichi Usami

\title{
Genetic features of hearing loss associated with ear anomalies: PDS and EYA1 mutation analysis
}

Received: January 16, 2001 / Accepted: June 12, 2001

\begin{abstract}
Mutation analysis of the $P D S$ gene and the $E Y A 1$ gene, which are reported to be responsible for hearing loss associated with ear anomalies, was performed in 24 deaf patients with various middle and inner ear anomalies. The present study was done to clarify the spectrum of middle and inner ear malformations covered by these two genes. $P D S$ mutations were found only in patients with enlarged vestibular aqueducts and $E Y A 1$ mutations were detected only in patients with ear pits and cervical fistulae, indicating that these two genes are associated with particular forms of middle and inner ear malformation. The genetic approach provides a strong tool for the diagnosis of hearing loss associated with ear anomalies.
\end{abstract}

Key words $P D S \cdot E Y A 1 \cdot$ Ear anomaly $\cdot$ Enlarged vestibular aqueduct $\cdot$ Mutation analysis

\section{Introduction}

Recent advances in computed tomography (CT) imaging techniques have provided us with detailed information regarding inner, middle, and outer ear malformations. Temporal bone CT is currently considered to be a useful examination to diagnose sensorineural hearing loss (SNHL), because approximately $20 \%$ of congenital SNHL patients are reported to have radiologically proven inner ear malformations (Jackler et al. 1987). Recent reports also

A. Namba $\cdot$ S. Abe $\cdot$ H. Shinkawa

Department of Otorhinolaryngology, Hirosaki University School of Medicine, Hirosaki, Japan

W.J. Kimberling

Department of Genetics, Boys Town National Research Hospital, Omaha, NE, USA

S. Usami $(\square)$

Department of Otorhinolaryngology, Shinshu University School of

Medicine, 3-1-1 Asahi, Matsumoto 390-8621, Japan

Tel. +81-263-37-2666; Fax +81-263-36-9164

e-mail: usami@hsp.md.shinshu-u.ac.jp support a high prevalence of inner ear anomalies among hearing loss patients. Woolford et al. (1995) reported $29.5 \%$ abnormal CT scans in adults and children awaiting cochlear implantation. Bamiou et al. (2000) found a $28.4 \%$ yield of abnormalities in the CT scans of SNHL patients.

Remarkable progress has been made in the molecular genetics of deafness in the past decade, specifically in the past few years, and discoveries about genes have greatly accelerated. More than 40 genes have so far been reported to be responsible for syndromic and nonsyndromic hearing loss (Van Camp G, Smith RJH. Hereditary Hearing Loss Homepage: http://dnalab-www.uia.ac.be/dnalab/hhh). Among them, two genes, $P D S$ and $E Y A 1$, were reported to be associated with inner, middle, or outer ear malformations. The $P D S$ gene was originally reported as being responsible for Pendred syndrome (MIM274600), an autosomal recessive disorder previously described as associated with deafness and goiter (Everett et al. 1997). However, the $P D S$ gene was later demonstrated to also cover the nonsyndromic hearing loss associated with enlarged vestibular aqueduct (EVA) (Usami et al. 1999b). These two recessive inherited diseases, previously considered distinct, are now known to be a continuum of diseases associated with EVA and caused by one gene. Thirty-eight mutations in $P D S$ that cause Pendred syndrome or nonsyndromic hearing loss associated with EVA have been reported (see the Human Gene Mutation Database at the Institute of Medical Genetics in Cardiff: http://archive.uwcm.ac.uk/uwcm/mg/hgmd0.html).

The EYA1 gene is responsible for branchio-oto-renal (BOR) syndrome (MIM113650) (Abdelhak et al. 1997), which is an autosomal dominant hereditary disease characterized by (1) branchial fistulae (cysts) and/or congenital preauricular fistulae; (2) anomalies of the pinnae, external, middle, and inner ears, accompanied by hearing loss; and (3) renal abnormalities. We have recently demonstrated that mutations in the PDS and EYA1 genes are found in hearing loss patients with ear anomalies (Usami et al. 1999a,b). Because the types of anomaly caused by these two genes overlap, PDS and EYA1 mutation analysis was performed to determine the spectrum of anomalies. 


\section{Subjects and methods}

Forty-four (25.4\%) of 173 congenital hearing loss patients who underwent $\mathrm{CT}$ scans were diagnosed with various types of inner and middle ear anomalies. Among them, 24 patients (10 male, 14 female) gave informed consent for participation in the mutation analysis project. From anamnestic evaluation, 10 patients had a family history of hearing loss (six dominant, four recessive) and 14 were sporadic cases (Table 1). Two patients were associated with cervical fistulae or ear pits (Table 1). Otherwise, no additional clinical manifestations, such as goiter, were found. All patients had been screened for the 1555 mitochondrial and GJB2 mutations, and none were detected. CT scans of the temporal bone were performed in $1.5-\mathrm{mm}$ serial axial cuts. Axial scans were oriented parallel to the infraorbital-meatal line. Mutation screening by DNA direct sequencing was performed as described elsewhere (Usami et al. 1999a,b).

In brief, intronic polymerase chain reaction (PCR) primers flanking each exon (Everett et al. 1997) were used to amplify exons 1-21 in the genomic DNA samples of the $P D S$ gene to detect mutations. For EYA1, the nucleotide sequence of the primers reported earlier (Abdelhak et al. 1997) were used for amplification of exons. The sequences of these primers are located in the flanking introns. Exons 1-16 of EYA1 were amplified from genomic DNA samples by PCR. PCR products were directly sequenced as described in previous papers (Kumar et al. 1998; Usami et al. 1999a). DNA samples from 96 unrelated Japanese individuals with normal hearing were used as a control group.

\section{Results}

Types of anomalies and detected mutations are summarized in Table 1. $P D S$ mutations were found in eight patients and EYA1 mutations in two patients. All of the detected mutations were segregated with deafness/anomaly in each family and were not found in 96 normal hearing control samples. Four missense and three frameshift mutations were found in the $P D S$ gene. Four patients were found to be compound heterozygous, and four were heterozygous with no observable second mutations. $P D S$ gene mutations were identified only in the patients with EVA. Eight of 14 cases with EVA (57.1\%) had PDS mutations. For the EYA1 mutations, a nonsense mutation in exon 7 and a missense mutation in exon 12 were noted in the patients with preauricular sinus or cervical fistulae.

\section{Discussion}

It has been reported that several types of syndromic and nonsyndromic hearing loss, including Pendred syndrome, BOR syndrome, Waardenburg's syndrome, DiGeorge's syndrome, Wildervanck syndrome, Fountain syndrome,
Treacher Collins syndrome, nonsyndromic hearing loss with EVA, and DFN3, are associated with various forms of ear anomalies (for review, see Smith and Harker 1998). This association indicates that many genes could be involved in the various types of anomalies in the external, middle, and inner ear. In the present study, $P D S$ and $E Y A 1$ gene mutations were found in $41.7 \%$ of cases with ear malformations, suggesting that these two genes are highly associated with hearing loss caused by ear anomalies. Seven of eight $P D S$ mutations were described in our previous papers (Usami et al. 1999a,b). The present paper further adds a novel PDS mutation, 1652insT. This mutation was segregated with the deafness/anomaly and was not found in the normal control samples, suggesting that it is a disease-causing mutation.

Four patients (\#1, 2,3, and 8 in Table 1 ) were found to be compound heterozygous, which is compatible with recessive properties of the PDS gene. However, another four patients (\#4, 5, 6, and 7 in Table 1) without apparent second mutations must have a second mutation in another allele. It is possible that the second mutation exists in the promoter region, which we did not analyze. It is unlikely that $P D S$ mutations have a dominant effect, because parents (of patients with EVA) who have heterozygous $P D S$ mutations did not have EVA. Caution is especially due when considering the parents of EVA patients who themselves have hearing loss (\#5 and 6 in Table 1). Although such parents had mild hearing loss, suggesting from the anamnestic evaluation that the condition was passed on by dominant inheritance, their audiological characteristics were different from those typically found in patients with EVA and their hearing impairment may be due to other causes.

The present study also revealed the spectrum of malformation caused by these two genes. EVA has been recognized as the single most common imaging abnormality in SNHL (Mafee et al. 1992). Of 14 patients with EVA, PDS mutations were found in $8(57.1 \%)$. The present results are in line with the recent report by Reardon et al. (2000), who observed a high frequency (33 of 43) of PDS gene mutations in singleton cases with EVA. It was also reported that 7 of 45 BOR syndrome patients had EVA (Chen et al. 1995). EVA has so far been reported in Pendred syndrome, nonsyndromic hearing loss associated with EVA (Usami et al. 1999b), and BOR syndrome (Chen et al. 1995). Therefore, EVA is recognized to be a genetically heterogenous phenotype. Despite the previously mentioned reports, we did not find EYAl mutations in the EVA cases studied here.

With regard to phenotypic variability caused by the $P D S$ gene, six of eight patients had an EVA malformation only. However, the other two patients had other inner and middle ear malformations in addition to EVA; one had hypoplastic cochlea and enlarged vestibule, and the other had hypoplastic cochlea and ossicular chain malformation. Pendred syndrome was sometimes found with Mondini malformation, which is considered by some investigators to be a more severe manifestation of EVA (Valvassori et al. 1978; Emmett et al. 1985; Johnsen et al. 1987; Mafee et al. 1992; Zalzal et al. 1995). The two cases that were found in this study support these previous reports. Although there 


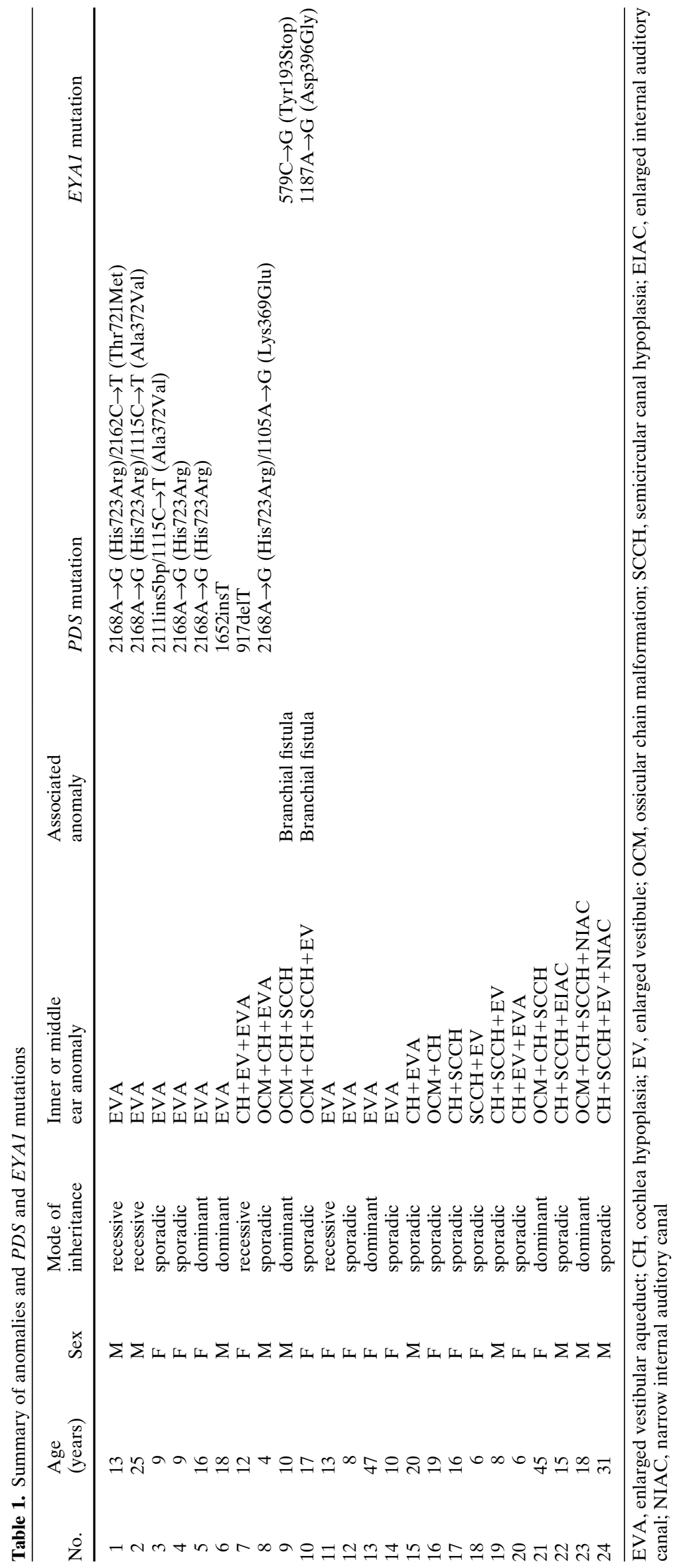


have been no reports of ossicular chain middle ear malformation concurrent with Pendred syndrome, it may be that the $P D S$ gene has an effect on middle ear malformations as well. It is also conceivable that other genes are involved in this phenotype.

In ten patients without EVA, two with characteristic diagnosis and observation of BOR syndrome, such as branchial fistulae and preauricular pits, had EYAl gene mutations. One of the EYA1 mutations, Y193X, had been reported previously (Usami et al. 1999a) and was dominantly inherited with phenotypes within the family (patient \#9 in Table 1). The other mutation, Asp396Gly, which is a novel missense mutation, was found in patient \#10 (Table $1)$. Interestingly, this patient is a sporadic case and both parents had no clinical symptoms or anomalies, nor did they have the EYAl mutation, suggesting this case may be a de novo mutation. There have been reports of BOR syndrome accompanied by inner, middle, and outer ear malformations (Fraser et al. 1978; Cremers and Noord 1980; Ng et al. 1989; Chen et al. 1995; Usami et al. 1999a). In the present study, we detected EYAl gene mutations in two patients with hypoplastic cochlea, semicircular canals hypoplasia, enlarged vestibule, and ossicular chain malformation. Our results confirm that middle and inner ear malformation accompanied by branchial fistulae and preauricular pits are caused by $E Y A 1$ gene mutations.

In six patients with EVA, and eight patients without EVA, branchial fistulae, or preauricular pits, neither gene mutation was detected. The present mutation screening did not include analysis of the upstream untranslated regions, which may be the sites of regulatory elements. Mutations in these regions may produce ear malformation or it may be that there is another, still unknown, locus that causes ear malformation. A large deletion is another possibility.

The present study clarified that, although there is some variability, there may be common features caused by the same gene. Classification based only on the inheritance mode is inaccurate because it is sometimes hampered by hearing loss due to various etiological factors. Future molecular genetic studies will determine the precise mechanisms of genetic influence on inner ear development, and ear anomalies will be classified on a genetic basis.

Acknowledgments We thank all of the families that participated in the present project. We would also like to thank Ms. C. Kawashima for technical assistance and Ms. A.C. Apple-Mathews for help in preparing the manuscript. This work is supported by the Ministry of Health and Welfare, Japan, (S.U.), and a Grant-in-Aid for Scientific Research from the Ministry of Education, Science and Culture of Japan (S.U.).

\section{References}

Abdelhak S, Kalatzis V, Heilig R, Compain S, Samson D, Vincent C, Weil D, Cruaud C, Sahly I, Leibovici M, Bitner-Glindzicz M, Francis M, Lacombe D, Vigneron J, Charachon R, Boven K, Bedbeder P,
Van Regemorter N, Weissenbach J, Petit C (1997) A human homologue of the Drosophila eyes absent gene underlies branchio-otorenal (BOR) syndrome and identifies a novel gene family. Nat Genet 15:157-164

Abe S, Usami S, Shinkawa H (1997) Three familial cases of hearing loss associated with enlargement of the vestibular aqueduct. Ann Otol Rhinol Laryngol 106:1063-1069

Bamiou DE, Phelps P, Sirimanna T (2000) Temporal bone computed tomography findings in bilateral sensorineural hearing loss. Arch Dis Child 82:257-260

Chen A, Francis M, Ni L, Cremers CW, Kimberling WJ, Sato Y, Phelps PD, Bellman SC, Wagner MJ, Pembrey M, Smith RJH (1995) Phenotypic manifestations of branchiootorenal syndrome. Am J Med Genet 58:365-370

Cremers CW, Fikkers-Van Noord M (1980) The earpits-deafness syndrome: clinical and genetic aspects. Int $\mathbf{J}$ Pediatr Otorhinolaryngol 2:309-322

Emmett JR (1985) The large vestibular aqueduct syndrome. Am J Otol 6:387-415

Everett LA, Glaser B, Beck JC, Idol JR, Buchs A, Heyman M, Adawi F, Hazani E, Nassir E, Baxevanis AD, Sheffield VC, Green ED (1997) Pendred syndrome is caused by mutations in a putative sulphate transporter gene (PDS). Nat Genet 17:411-422

Fraser FC, Ling D, Clogg D, Nogrady B (1978) Genetic aspects of the BOR syndrome: branchial fistulas, ear pits, hearing loss, and renal anomalies. Am J Med Genet 2:241-252

Jackler RK, Luxford WM, House WF (1987) Congenital malformations of the inner ear: a classification based on embryogenesis. Laryngoscope 97:2-14

Johnsen T, Larsen C, Friis J, Hougaard-Jensen F (1987) Pendred's syndrome. Acoustic, vestibular and radiological findings in 17 unrelated patients. J Laryngol Otol 101:1187-1192

Kumar S, Kimberling WJ, Weston MD, Schaefer BG, Berg MA, Marres HA, Cremers CW (1998) Identification of three novel mutations in human EYA1 protein associated with branchio-oto-renal syndrome. Hum Mutat 11:443-449

Mafee MF, Charletta D, Kumar A, Belmont H (1992) Large vestibular aqueduct and congenital sensorineural hearing loss. Am J Neuroradiol 13:805-819

Ng YY, Bellman S, Phelps PD (1989) Computed tomography of earpits-deafness syndrome. Br J Radiol 62:947-949

Reardon W, O'Mahoney CF, Trembath R, Jan H, Phelps PD (2000) Enlarged vestibular aqueduct: a radiological marker of Pendred syndrome, and mutation of the PDS gene. QJM 93:99-104

Smith SD, Harker LA (1998) Single gene influences on radiologicallydetectable malformations of the inner ear. J Commun Disord 31:391-408

Usami S, Abe S, Shinkawa H, Deffenbacher K, Kumar S, Kimberling WJ (1999a) EYA1 nonsense mutation in a Japanese branchio-otorenal syndrome family. J Hum Genet 44:261-265

Usami S, Abe S, Weston MD, Shinkawa H, Van Camp G, Kimberling WJ (1999b) Non-syndromic hearing loss associated with enlarged vestibular aqueduct is caused by PDS mutations. Hum Genet 104:188-192

Valvassori GE, Clemis JD (1978) The large vestibular aqueduct syndrome. Laryngoscope 88:723-728

Woolford TJ, Roberts GR, Hartley C, Ramsden RT (1995) Etiology of hearing loss and cochlear computed tomography: findings in preimplant assessment. Ann Otol Rhinol Laryngol Suppl 166:201206

Zalzal GH, Tomaski SM, Vezina LG, Bjornsti P, Grundfast KM (1995) Enlarged vestibular aqueduct and sensorineural hearing loss in childhood. Arch Otolaryngol Head Neck Surg 121:23-28 